\title{
Optical pattern recognition based on color vision models
}

\author{
M. S. Millán and M. Corbalán \\ Departamento de Optica y Departamento Física e Ingeniería Nuclear, Universidad Politécnica de Cataluña, \\ Campus Terrassa, E-08222 Terrassa, Spain
}

J. Romero

Departamento de Optica, Universidad de Granada, Fuentenueva s/n, 18071 Granada, Spain

\author{
M. J. Yzuel
}

Departamento de Física, Universidad Autónoma de Barcelona, E-08193 Bellaterra, Spain

Received May 2, 1995

\begin{abstract}
A channel transformation based on opponent-color theory of the color vision models is applied to optical pattern recognition so that the conventional red, green, and blue (RGB) channels are transformed into bright-dark, red-green, and yellow-blue (ATD) channels. Matched filtering and correlation are performed over the new components of the target and the scene in the ATD system. The proposed transformation allows us to reduce the number of channels commonly used in color pattern recognition, passing from the three RGB channels to the two red-green and yellow-blue opponent-color channels. (C) 1995 Optical Society of America
\end{abstract}

The introduction of color information in pattern recognition is especially useful when the contours and the intensity distribution do not provide enough information to permit the discrimination of images. The most extensive way to incorporate color into pattern recognition is through a trichromatic decomposition of the image into red, green, and blue (RGB) components that are independently analyzed. The RGB multichannel decomposition has been associated with matched filtering and optical correlation techniques for the performance of optical color pattern recognition. One of the methods used for the RGB decomposition is the illumination of a color transparency containing the target by three monochromatic wavelengths separately. ${ }^{1,2}$ The three wavelengths, belonging to the red, green, and blue regions, must be distributed in the visible spectrum. For the illumination of the optical correlator it has been proposed that the same wavelengths be used for the generation of the filters ${ }^{2}$ or that a white-light source combined with a grating in the scene plane be used. ${ }^{3} \quad$ Another method used for RGB decomposition is the acquisition and digitization of the image by a color camera. In this case the channels are defined by the response of the camera in a wide bandwidth. The RGB components of the scene are displayed in the correlator either on an achromatic spatial light modulator ${ }^{3}$ or on a color spatial light modulator. ${ }^{4}$ This method permits digital preprocessing of the acquired image before it is displayed.

Different problems in polychromatic pattern recognition have been studied in Refs. 1-6. The most common problem is the recognition of a given object, taking into account both the shape and the color distribution. ${ }^{1-4}$ Other problems are the recognition of a given shape regardless of the color distribution of the object and the identification of the color of the diverse zones that compose the object., ${ }^{5,6}$ Millán et al. ${ }^{5}$ proposed a recognition strategy that involves the signals used to generate the matched filters and the decision criteria applied to the RGB multichannel correlation results. Yamaba and Miyake ${ }^{6}$ proposed a transformation of the RGB components provided by the camera that considers the Hurvich-Jameson color vision $\operatorname{model}^{7}$ with some modifications. Their recognition system is based on the projected weight values obtained from a learning process by a neural board.

Color human vision models based on the opponentcolor theory define three channels from the RGB signals provided by the retinal receptors: the bright-dark luminance channel, the red-green opponent channel, and the yellow-blue opponent channel (ATD). In this Letter we consider the application of such models to color pattern recognition. The luminance channel is similar to a black-and-white channel that processes the intensity information of the scene in a conventional achromatic recognition system. When the intensity information is not enough for us to recognize the target without false alarms, we study the feasibility of using the two opponent-color channels to improve and achieve discrimination.

Among the diverse linear color vision models based on the opponent-color theory, we have focused our attention on the two updated ATD models proposed by Guth et al. ${ }^{8}$ and later by Boynton. ${ }^{9}$ The ATD models consist of an achromatic (A) bright-dark channel that may be regarded as the luminance channel, a $\mathrm{T}$ channel (tritan channel) that corresponds to the opponent response red-green, and a D channel (deutan channel) that corresponds to the opponent response yellow-blue. The model of Guth et al. is represented by the matrix equation

$$
\left[\begin{array}{l}
\mathrm{A} \\
\mathrm{T} \\
\mathrm{D}
\end{array}\right]=\left[\begin{array}{rcc}
0.5967 & 0.3654 & 0 \\
0.9553 & -1.2836 & 0 \\
-0.0248 & 0 & 0.0483
\end{array}\right]\left[\begin{array}{l}
\mathrm{R} \\
\mathrm{G} \\
\mathrm{B}
\end{array}\right],
$$



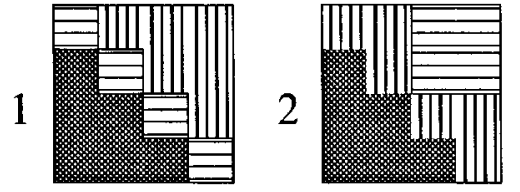

IIII Orange
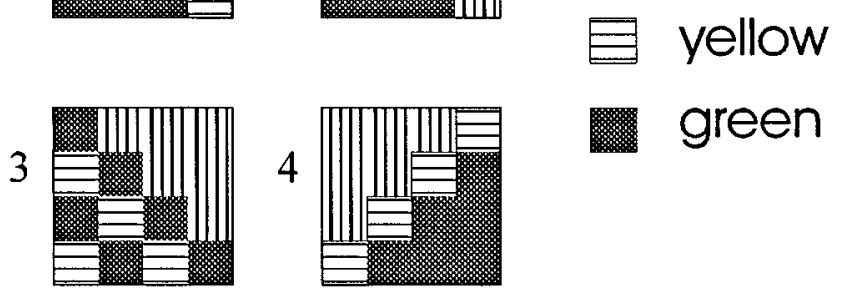

- green

Fig. 1. Polychromatic test. Object 1 is the target.

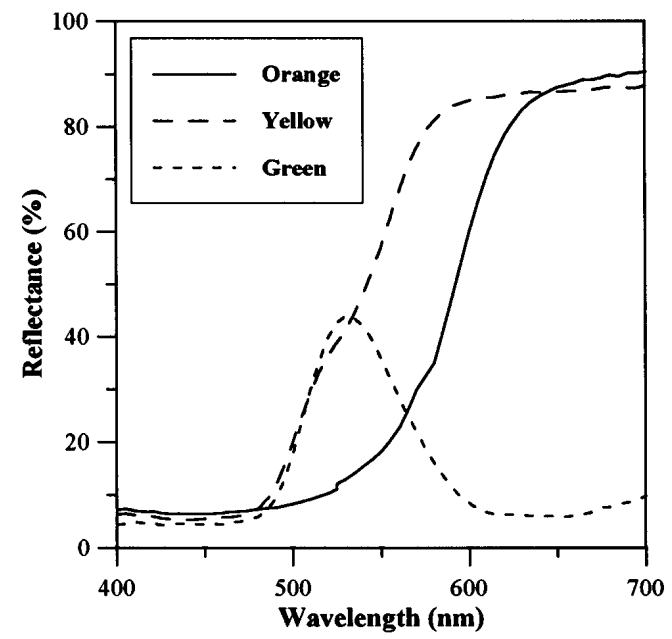

Fig. 2. Spectral reflectances of the colors of the test.

and Boynton's model is represented by the matrix equation

$$
\left[\begin{array}{l}
\mathrm{A} \\
\mathrm{T} \\
\mathrm{D}
\end{array}\right]=\left[\begin{array}{rrr}
1 & 1 & 0 \\
1 & -2 & 0 \\
1 & 1 & -1
\end{array}\right]\left[\begin{array}{l}
\mathrm{R} \\
\mathrm{G} \\
\mathrm{B}
\end{array}\right],
$$

where $R, G$, and $B$ are the normalized values of the human receptors according to Smith-Pokorny human spectral sensitivity curves. ${ }^{10}$ In our proposal we replace these values with those provided by the color camera in its corresponding RGB channels. Although the color camera has wide-bandpass spectral sensitivity curves, they overlap less than human R and $G$ curves. The transformation of the RGB channels into the ATD channels described by Eqs. (1) and (2) is to be applied as a preprocessing of the RGB image components provided by the acquisition system.

To illustrate the possibilities of the application of the ATD model to color pattern recognition we consider the polychromatic test of Fig. 1, where object 1 is the target. The different color zones (orange, yellow, and green) correspond to the spectral reflectances of Fig. 2. As a first step we use a black-and-white CCD camera to capture the test scene illuminated by fluorescent light of $5000 \mathrm{~K}$. The mean numerical values of the color zones and the black background of the digital image are given in Table 1. Using this image matrix, we perform a numerical correlation experiment in which we use a phase-only filter ${ }^{11}$ matched to object 1 and obtain the correlation results
(Table 2) corresponding to this conventional singlechannel recognition process. Taking a threshold value of $50 \%$ of the maximum correlation intensity value (peaks exceeding the threshold are boldfaced in Table 2) we note that objects 1 and 2 would be identified with the target, giving one false alarm for object 2. The results obtained in this achromatic channel prove that the introduction of color information is essential for solving our recognition problem. Then we use a 3CCD color camera (Sony DXC-930P) to capture the test scene in the illuminating conditions described above. Table 1 contains the mean numerical values of the diverse color zones and the black background in the digital RGB image components. We perform numerical correlation experiments, applying the multichannel recognition process described in Ref. 2 and taking into account the RGB channel system and the ATD channel system of the models of both Guth et al. and Boynton. Phase-only matched filtering is used in every case. The correlation is performed in each channel separately, and a threshold of $50 \%$ of the maximum is applied. The results are shown in Table 3 , in which the peaks that exceed the threshold value in each channel are boldfaced.

The decision rule in the RGB channel system is represented by the logical operator AND applied to the boldfaced peaks of the three channels. In this RGB channel system object 1 is recognized without false alarms. This confirms that the discrimination capability of the recognition system highly increases when color content is introduced.

The correlation results obtained for the ATD channel systems lead to similar conclusions for the models of Guth et al. and Boynton, although the numerical values of the correlation intensities do not coincide exactly. For our proposal we could point out that the rather qualitative description of the Boynton model offers a simpler method to follow than the

Table 1. Mean Numerical Values of the Color Zones in the Digital Images of the Test Scene

\begin{tabular}{lccccc}
\hline & & \multicolumn{4}{c}{ Color Zone } \\
\cline { 3 - 6 } Camera & Channel & Orange & Yellow & Green & Black \\
\hline $\begin{array}{l}\text { Black and } \\
\text { white }\end{array}$ & Intensity & 155 & 195 & 125 & 60 \\
Color & $\mathrm{R}$ & 227 & 229 & 72 & 0 \\
& $\mathrm{G}$ & 76 & 222 & 214 & 0 \\
& $\mathrm{~B}$ & 6 & 8 & 0 & 0 \\
\hline
\end{tabular}

Table 2. Correlation Results for the Recognition of Object 1 with an Achromatic Single Channel ${ }^{a}$

\begin{tabular}{lccccc}
\hline & \multicolumn{5}{c}{ Correlation Intensity Values } \\
\cline { 2 - 5 } & Obj. & Obj. & Obj. & Obj. & Threshold \\
$\left(\times 10^{5}\right)$
\end{tabular}

${ }^{a}$ Boldface indicates peaks exceeding threshold. 
Table 3. Correlation Results for the Recognition of Object 1 with Color Channel Systems ${ }^{a}$

\begin{tabular}{|c|c|c|c|c|c|}
\hline \multirow[b]{2}{*}{ Channel System } & \multicolumn{4}{|c|}{ Correlation Intensity Values } & \multirow[b]{2}{*}{ Threshold } \\
\hline & Obj. 1 & Obj. 2 & Obj. 3 & Obj. 4 & \\
\hline $\mathrm{R}$ & $1.59 \times 10^{6}$ & $1.57 \times 10^{6}$ & $0.39 \times 10^{6}$ & $0.56 \times 10^{6}$ & $0.79 \times 10^{6}$ \\
\hline $\mathrm{G}$ & $1.49 \times 10^{6}$ & $0.42 \times 10^{6}$ & $1.44 \times 10^{6}$ & $0.49 \times 10^{6}$ & $0.74 \times 10^{6}$ \\
\hline B & $1.96 \times 10^{3}$ & $1.51 \times 10^{3}$ & $0.64 \times 10^{3}$ & $0.20 \times 10^{3}$ & $0.98 \times 10^{3}$ \\
\hline Recognition (AND) & Yes & No & No & No & \\
\hline \multicolumn{6}{|l|}{ Guth et $a l .^{b}$} \\
\hline $\mathrm{A}$ & $1.12 \times 10^{6}$ & $0.79 \times 10^{6}$ & $0.36 \times 10^{6}$ & $0.60 \times 10^{6}$ & $0.56 \times 10^{6}$ \\
\hline $\mathrm{T}$ & $1.43 \times 10^{6}$ & $0.55 \times 10^{6}$ & $0.89 \times 10^{6}$ & $0.19 \times 10^{6}$ & $0.72 \times 10^{6}$ \\
\hline $\mathrm{D}$ & $8.62 \times 10^{2}$ & $8.57 \times 10^{2}$ & $2.10 \times 10^{2}$ & $3.27 \times 10^{2}$ & $4.31 \times 10^{2}$ \\
\hline Recognition (AND) & Yes & No & No & No & \\
\hline \multicolumn{6}{|l|}{ Boynton $^{c}$} \\
\hline $\mathrm{A}$ & $4.68 \times 10^{6}$ & $2.70 \times 10^{6}$ & $2.06 \times 10^{6}$ & $2.60 \times 10^{6}$ & $2.34 \times 10^{6}$ \\
\hline $\mathrm{T}$ & $3.73 \times 10^{6}$ & $1.29 \times 10^{6}$ & $3.61 \times 10^{6}$ & $0.58 \times 10^{6}$ & $1.86 \times 10^{6}$ \\
\hline $\mathrm{D}$ & $4.52 \times 10^{6}$ & $2.60 \times 10^{6}$ & $2.09 \times 10^{6}$ & $2.55 \times 10^{6}$ & $2.26 \times 10^{6}$ \\
\hline Recognition (AND) & Yes & No & No & No & \\
\hline
\end{tabular}

${ }^{a}$ Boldface indicates peaks exceeding threshold.

${ }^{b}$ Ref. 8.

${ }^{c}$ Ref. 9 .

model of Guth et al., which was obtained specifically for human vision. We analyze separately the results obtained in the A channel and the results obtained in the subset of $\mathrm{T}$ and $\mathrm{D}$ channels. If we consider only the A channel of luminance, objects 1,2 , and 4 are identified with the target. So, as in the previous case with a black-and-white CCD camera, false alarms are produced in the A channel. On the other hand, we observe that the use of the two T and D opponent-color channels can provide enough discrimination capability for one to recognize object 1 without false alarms. Applying the operator AND to the correlation peaks higher than the threshold in both $\mathrm{T}$ and $\mathrm{D}$ channels, we achieve the recognition of object 1 . To solve color recognition problems where color information is particularly necessary, either the RGB channel system or the TD channel subsystem can be applied. In this second case the use of the TD channels has the main advantage of concentrating the color information contained in the three RGB channels in only two channels, so that the recognition process of color objects can be considerably simplified.

The study has also been carried out for scenes whose colors cover a wide range of hue in the visible spectrum (magenta, blue, green, yellow, red, etc.). The results obtained in all cases are similar to those presented above. Sometimes, when all the colors have a similar hue, the number of channels can be further reduced to only one. However, for a general case of color recognition the whole TD subsystem should be considered.

In conclusion, significant advantages in color pattern recognition can be obtained by application of a transformation of the multichannel system, according to the color vision models based on the opponent-color theory. We have considered the models proposed by Guth et al. and Boynton to pass from the RGB system to the ATD system, and both of them have led us to similar recognition results in the test scenes that we have analyzed. The discrimination capability obtained in the A channel is similar to that obtained in a conventional achromatic channel when only the intensity information of the scene is considered. The discrimination capability obtained with the RGB trichannel system for the recognition of polychromatic objects has been also obtained with the subsystem of only two $\mathrm{T}$ and $\mathrm{D}$ channels.

This research has been financed by Comisión Interministerial de Ciencia y Tecnología projects TAP930667-C03-01 and TAP93-0667-C03-02.

\section{References}

1. F. T. S. Yu, Opt. Eng. 23, 690 (1984).

2. M. S. Millán, J. Campos, C. Ferreira, and M. J. Yzuel, Opt. Commun. 73, 277 (1989).

3. F. T. S. Yu and B. Javidi, Opt. Commun. 56, 384 (1986).

4. Z. Q. Wang, C. M. Cartwright, C. Souter, and W. A. Gillespie, Appl. Opt. 32, 715 (1993).

5. M. S. Millán, M. J. Yzuel, J. Campos, and C. Ferreira, Appl. Opt. 31, 2560 (1992).

6. K. Yamaba and Y. Miyake, Opt. Eng. 32, 33 (1993).

7. L. M. Hurvich and D. Jamenson, Psychol. Rev. 64, 384 (1957).

8. S. L. Guth, R. W. Massof, and T. Benzschawel, J. Opt. Soc. Am. 70, 197 (1980).

9. R. M. Boynton, Color Res. Appl. 11, 244 (1986).

10. V. C. Smith and J. Pokorny, Vision Res. 15, 161 (1975).

11. J. L. Horner and P. D. Gianino, Appl. Opt. 23, 812 (1984). 